\title{
Trends and In-Hospital Outcomes of Splanchnic Vein Thrombosis Associated with Gastrointestinal Malignancies: A Nationwide Analysis
}

\author{
Shivani Handa ${ }^{a} \quad K^{2}$ amesh Gupta ${ }^{b} \quad$ Michelle Sterpi $^{a} \quad$ Ahmad Khan $^{c}$ \\ Abhinav Hoskote $^{\mathrm{a}}$ Anup Kasi ${ }^{\mathrm{d}}$ \\ aDepartment of Internal Medicine, Icahn School of Medicine at Mount Sinai, Mount Sinai West and Morningside, \\ New York, NY, USA; 'D Department of Internal Medicine, UMMS-Baystate Medical Center, Springfield, MA, USA; \\ 'Department of Internal Medicine, West Virginia University-Charleston Area Medical Center, Charleston, WV, USA; \\ ${ }^{\mathrm{d} D e p a r t m e n t}$ of Oncology, Kansas University Medical Center, Kansas City, KS, USA
}

\section{Keywords}

Splanchnic vein thrombosis · Portal vein thrombosis · BuddChiari syndrome $\cdot$ Pancreatic cancer $\cdot$ Hepatobiliary cancer

\begin{abstract}
Introduction: Gastrointestinal cancers have a strong association with splanchnic vein thrombosis (SVT), yet the hospitalization data is unknown. Objective and Methods: We analyzed around 78 million discharges from the 2007-2017 Nationwide Inpatient Sample with an inclusion criterion of adult patients admitted for portal or hepatic vein thrombosis as a primary diagnosis with a gastrointestinal or hepatobiliary malignancy as a secondary diagnosis. The outcomes were in-hospital mortality, complication rates, and resource utilization. Odds ratios (OR) and means were adjusted for confounders using multivariate regression analysis models. Results: Out of the total 32,324 hospitalizations for SVT, $3,220(10 \%)$ were associated with a GI malignancy, of which hepatocellular carcinoma (HCC) and pancreatic cancer were the most common. Portal vein thrombosis accounted for $95 \%$ of these hospitalizations. Admissions for pancreatic cancer-associated SVT have increased by 7.2 times from 2007 to 2017. Patients with SVT and concomitant GI malig-
\end{abstract}

karger@karger.com www.karger.com/gat

Karger"

BOPEN ACCESS
(C) 2021 The Author(s)

Published by S. Karger AG, Basel

This article is licensed under the Creative Commons AttributionNonCommercial-NoDerivatives 4.0 International License (CC BYNC-ND) (http://www.karger.com/Services/OpenAccessLicense) Usage and distribution for commercial purposes as well as any distribution of modified material requires written permission. nancies were significantly older and had a higher comorbidity score than those with SVT without GI malignancy. Risk of inpatient mortality for SVT patients were significantly higher for patients with gastric cancer (rate: $12.1 \%, \mathrm{OR} 8.6,95 \% \mathrm{Cl}$ : 1.8-39.7) and HCC (rate: $7.6 \%$, OR 2.77, 95\% Cl 1.5-4.8) as compared to non-GI malignancy-related SVT. Odds of variceal bleeding were significantly higher for patients with HCC (OR 1.67, 95\% Cl: 1.2-2.34) than patients without GI malignancy. Conclusions: Digestive cancer-associated SVTs constitute $10 \%$ of all SVT related hospitalizations and are significantly increasing in the past decade. We report the baseline characteristics and predictors of inpatient mortality in this study.

(c) 2021 The Author(s).

Published by S. Karger AG, Basel

\section{Introduction}

Venous thromboembolism in the splanchnic circulation including the portal, hepatic, splenic, or mesenteric veins is a rare clinical entity. However, a postmortem study from Sweden pegged the prevalence of portal vein thrombosis (PVT) alone at 1\% [1-3]. Thrombotic complications are more common in patients with cancer, pre-

Shivani Handa

Department of Internal Medicine, Mount Sinai West and Morningside 1000 Tenth Avenue

New York, NY 10019 (USA)

shivanihanda5@gmail.com 
sumably due to increased levels of leukocytes, platelets, and tissue factor-positive microvesicles [4]. The fundamental components of Virchow's triad, including hypercoagulability, endothelial dysfunction (by tumor invasion or release of inflammatory cytokines), and static blood flow (e.g., direct impaction resulting from a malignant mass or organomegaly) function synchronously in the genesis of malignancy-associated splanchnic vein thrombosis (SVT) [1].

Digestive cancers account for $18.5 \%$ of all cancers and have a strong association with the occurrence of PVT [5]. Hepatocellular carcinoma (HCC), is the commonest malignancy underlying PVT, with a prevalence of around $35-50 \%$ in patients with cirrhosis complicated by HCC [6]. Furthermore, the presence of PVT is considered a strong negative prognostic factor due to the increased risk of spreading cancer cells into the systemic circulation, leading to a high recurrence risk. For this reason, it is considered a contraindication to liver transplant [7]. Other digestive cancers are also commonly seen in PVT. Ogren et al. [3] in a postmortem study of over 23,000 autopsies demonstrated PVT to be present as frequently as 1 per 100 autopsies, of which $44 \%$ were associated with secondary hepatobiliary and GI cancers other than HCC. Pancreatic cancer is a thrombogenic malignancy [8] with an incidence of venous thromboembolism between 10 and $60 \%[9,10]$. Almost half of the venous thrombosis in patients with pancreatic cancer occurs in the splanchnic circulation $[11,12]$.

The current incidence and the healthcare burden of SVT is not well known. Hitherto, there are no data regarding morbidity or mortality of patients with digestive cancer-associated SVT, mainly PVT. The objective of this nationwide, multi-hospital study was to determine the rates of hospitalization and outcome of patients with SVT associated with GI malignancies. We aimed to determine the type of digestive cancer and the other baseline characteristics of patients with SVT that correspond to a higher in-hospital mortality.

\section{Methods}

\section{Data Acquisition}

We performed a retrospective, multicenter, observational study using data from the Nationwide Inpatient Sample (NIS). The NIS is managed by the Healthcare Cost and Utilization Project and regulated by the Agency for Healthcare Research and Quality. It is an administrative database composed of roughly $20 \%$ discharges from all US hospitals. The database provides information on discharge weights which can then be used to extrapolate the data to derive the national rates [13].
The NIS database provides information regarding the index hospital admission and includes patient demographic data, primary and secondary diagnosis, procedures, hospital characteristics, and inpatient and discharge mortality rates. Each record includes 1 primary and up to 24 secondary discharge diagnoses, procedure codes, demographic data, hospitalized inpatient mortality indicator, payer status, and various demographic data points.

\section{Variables}

We identified all adult patients with a primary discharge diagnosis of PVT (ICD 9: 452; ICD 10: I81) or hepatic vein thrombosis/ Budd-Chiari syndrome (ICD 9: 453.0, ICD 10: I82.0) along with gastrointestinal malignancy as a secondary diagnosis (esophageal, gastric, hepatic, pancreatic, colon, or cholangiocarcinoma). All ICD codes used in the study are enlisted in online suppl. Table 1; for all online suppl. material, see www.karger.com/doi/ $10.1159 / 000513368$. Other types of SVT, that is, splenic vein and mesenteric vein thrombosis currently do not have a specific ICD 9 or ICD 10 codes. Thus, the term SVT pertains to portal and hepatic vein thrombosis only in this study. We excluded patients with a potential alternative etiology of SVT such as polycythemia vera, primary myelofibrosis, and essential thrombocythemia from the SVT in GI malignancy group. The aim of the study was to characterize the rate of hospitalization, compare the baseline characteristics and in-hospital outcomes for SVT associated with different types of GI malignancies as compared to SVT without GI malignancies over the study period. NIS provides length of stay and total hospitalization charges for each hospitalization. Similarly, other baseline characteristics studied as potential confounders were age, sex, race/ethnicity, insurance status, and comorbidity burden encoded as Charlson comorbidity index. Several hospital characteristics were studied as well including location (rural or urban), hospital teaching status, and hospital bed size (divided into $<250$, $250-400$ and $>400$ ).

\section{Statistical Analysis}

Baseline characteristics were compared between GI malignancy and non-GI malignancy hospitalizations using either a $\chi^{2}$ test (for categorical variables $>5$ in value), Fisher's exact test (for categorical variables $\leq 5$ in value), or a Student's $t$ test for continuous variables (Table 1). Univariate and multivariate logistic regression analysis was used to determine factors associated with higher inpatient mortality (Table 2). Multivariable logistic regression analysis was used for assessing inpatient mortality data to adjust for potential confounders such as age, sex, race, median income, Charlson comorbidity index, insurance status, hospital size, teaching status, prolonged ventilation, or shock (Table 3). Linear regression model was used for analyzing the outcomes of hospitalization charges and length of stay (these are represented in Table 3 along with beta-coefficients). In-hospital complications, specifically hepatic encephalopathy, ascites, variceal bleeding, requirement for blood transfusion, and mesenteric ischemia were compared between each major malignancy (hepatic cancer, pancreatic cancer, and colon cancer) and the rest of the hospitalizations for SVT. Univariate logistic regression analysis was used for analyzing inhospital complication rates. Unadjusted odds ratio for these are reported in Table 4. All analyses were performed using STATA 15.0. 


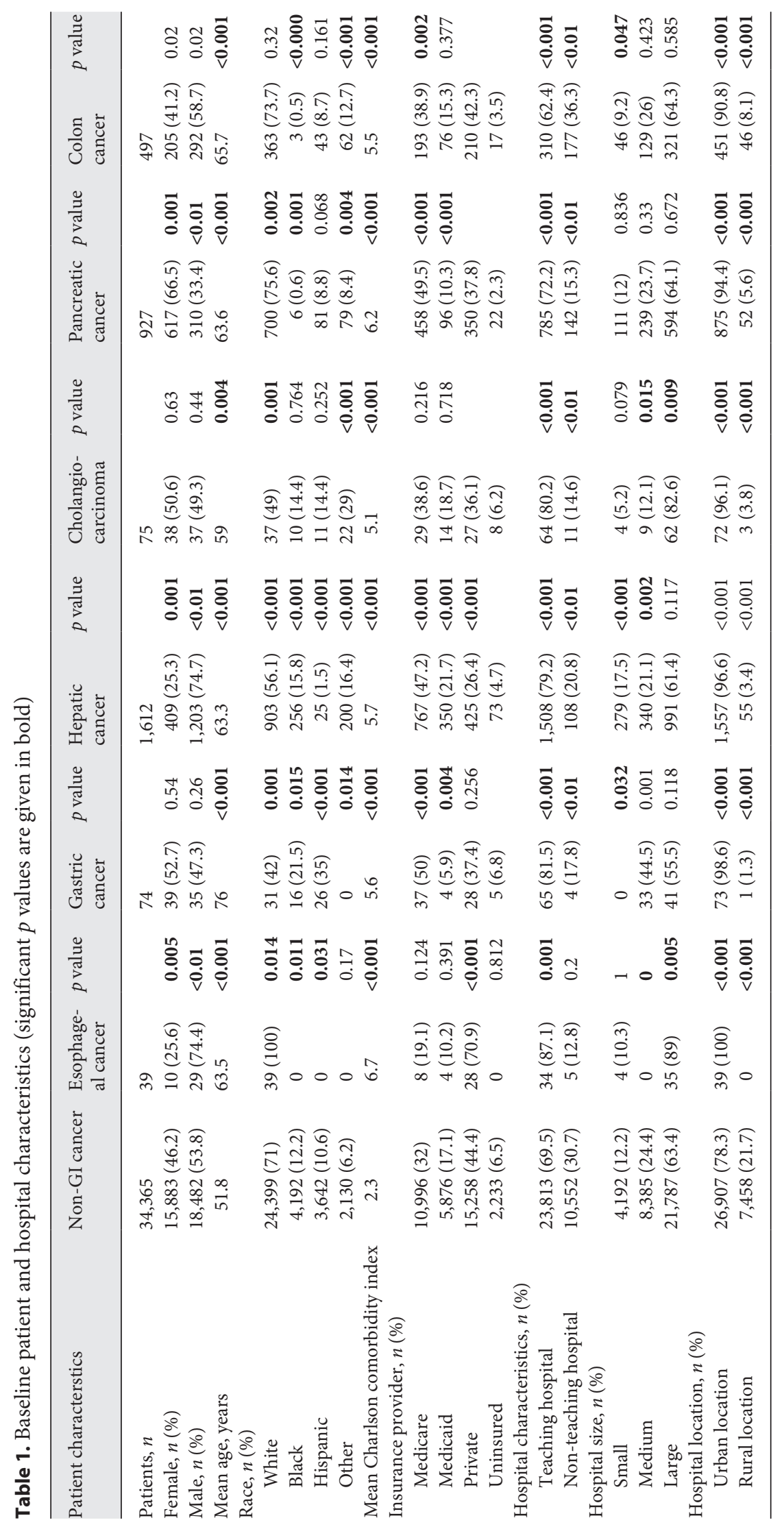


Table 2. Univariate and multivariate regression analysis of factors associated with inpatient mortality

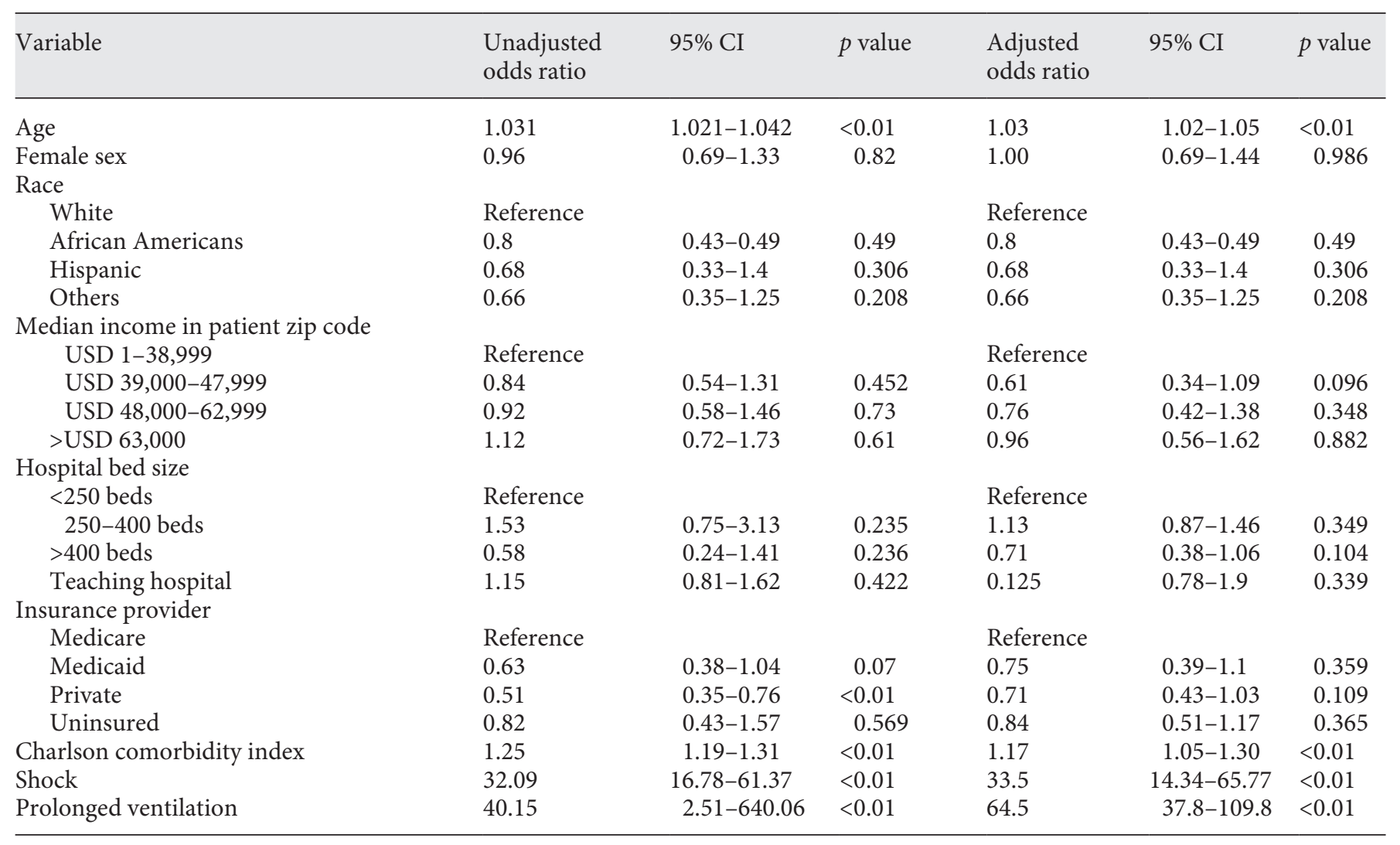

\section{Results}

From 2007 to 2017, NIS contained 32,324 weighted admissions with a principal discharge diagnosis of SVT, out of which 3,220 (10\%) were identified in patients with GI malignancies. The rates of SVT-related hospitalizations were highest among patients with hepatic cancer (5.0\%), which accounted for half of all admissions followed by pancreatic cancer $(2.9 \%)$, colorectal cancer ( $1.6 \%)$, cholangiocarcinoma $(0.2 \%)$, gastric cancer $(0.2 \%)$, and esophageal cancer $(0.1 \%)$. PVT was much more common than Budd-Chiari syndrome, accounting for $95.3 \%$ of all SVTs $(N=3,068)$. Hospitalizations for SVT related to GI malignancies have increased by 4.5 times from 2007 to 2017 as compared to admissions for SVT related to other causes which have increased by 3.2 times over the span of a decade ( $p$ value $<0.01$ ). Among patients with GI malignancies, hospitalizations for SVT in pancreatic cancer have the highest rise in trend, increasing by 7.2 times in 11 years, whereas the trend has been relatively stable in patients with esophageal, gastric and cholangiocarcino- ma. Trends for SVT-related hospitalizations in various GI malignancies are depicted in Figure 1. Corresponding trends for yearly incidence of GI malignancies are shown in Figure 2.

\section{Baseline Characteristics}

We compared the baseline characteristics of patients hospitalized for SVT related to GI cancers with those without GI cancers. Patients with esophageal and hepatic cancers admitted with SVT had significantly higher percentages of males, whereas pancreatic cancer patients had a higher proportion of females. The mean age was significantly higher for all patients with underlying GI cancers and was highest for gastric cancer (76 years, $p<0.001$ ). White patients were significantly higher in esophageal and pancreatic cancer groups but lower in gastric, hepatic, and cholangiocarcinoma. As expected, all GI malignancies had significantly higher proportions of patients with $>3$ Charlson comorbidity scores. Patients with gastric, hepatic, and pancreatic cancer had a significantly higher proportion of Medicare ben- 


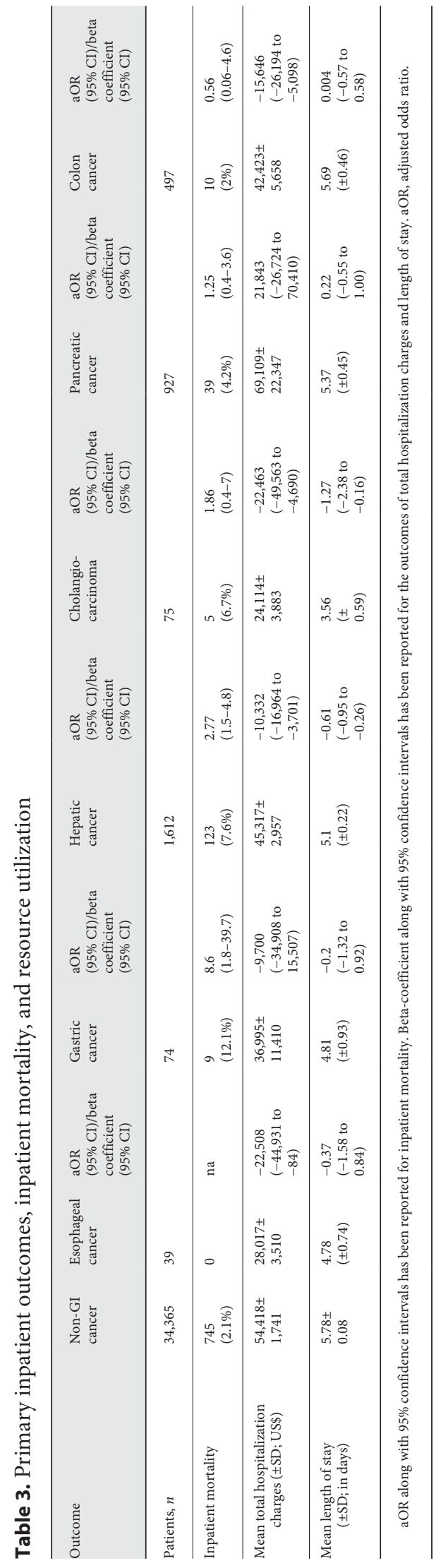

Splanchnic Vein Thrombosis in Gastrointestinal Malignancies

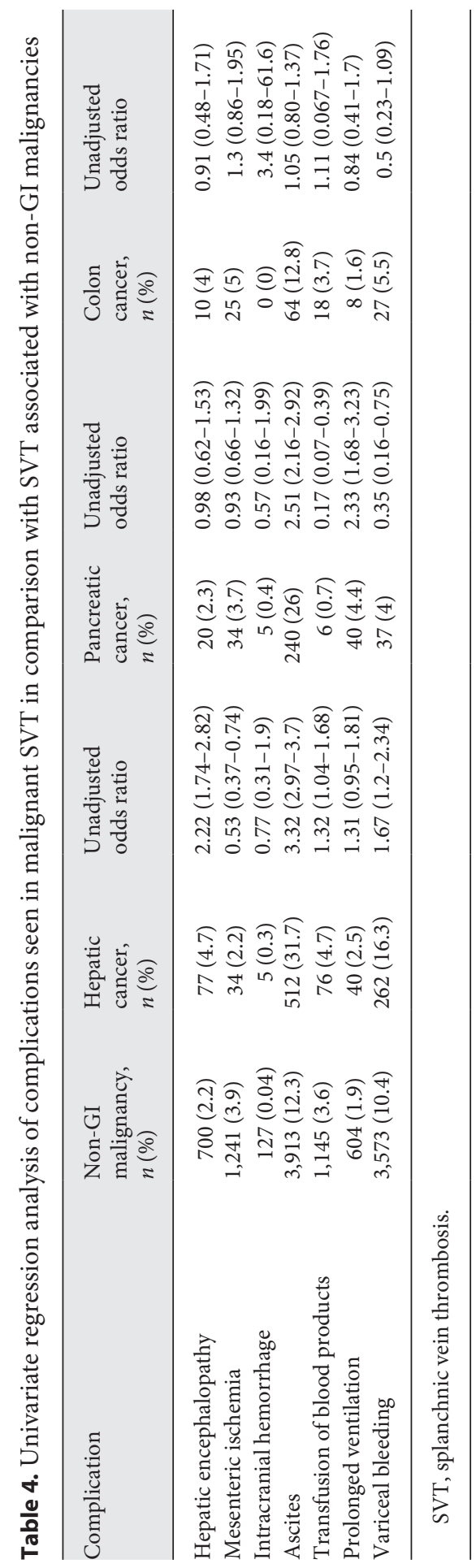

Gastrointest Tumors 2021;8:71-80 


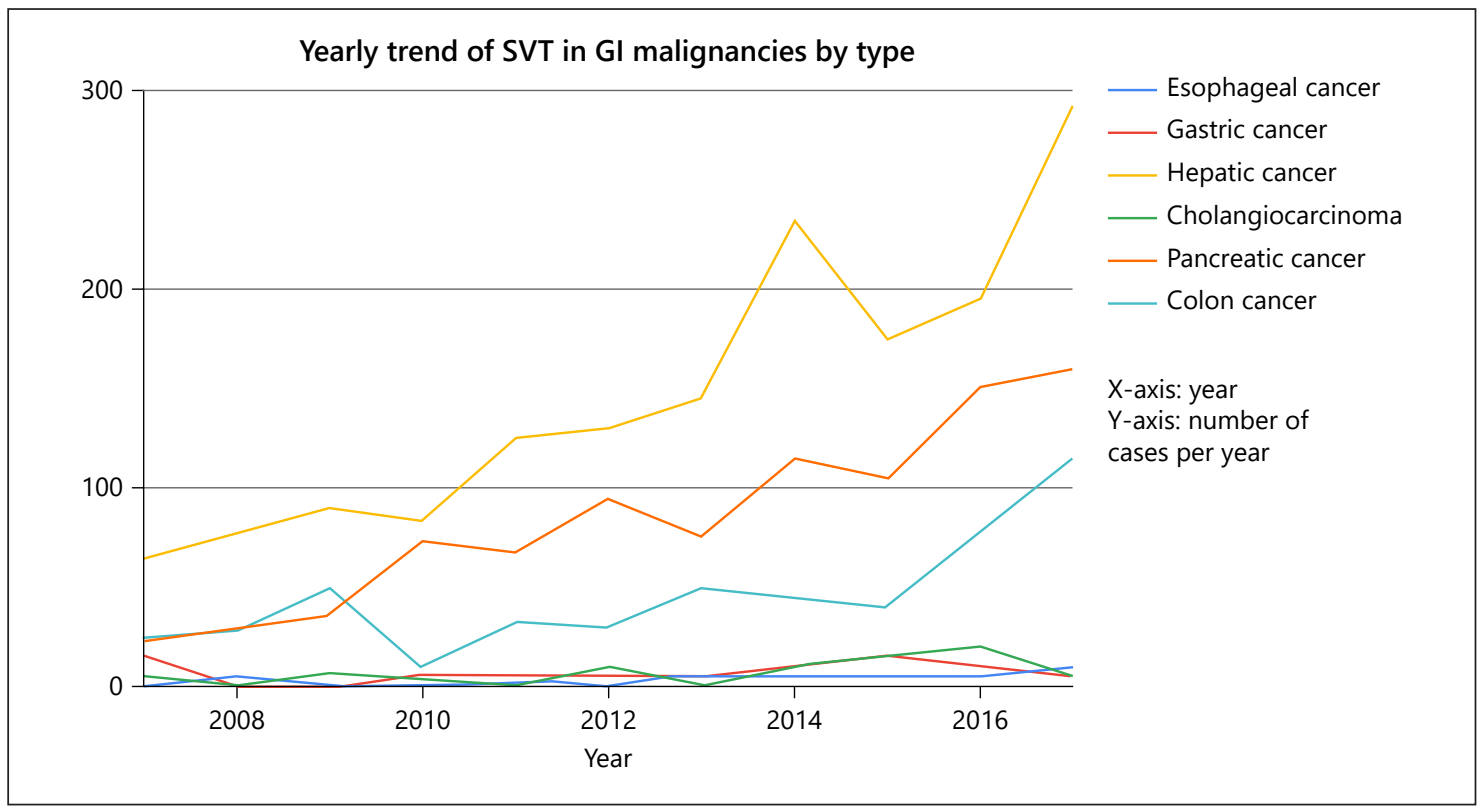

Fig. 1. Yearly trend of hospitalizations for SVT in GI malignancies compared by the type of malignancy from 2007 to 2017. SVT, splanchnic vein thrombosis.

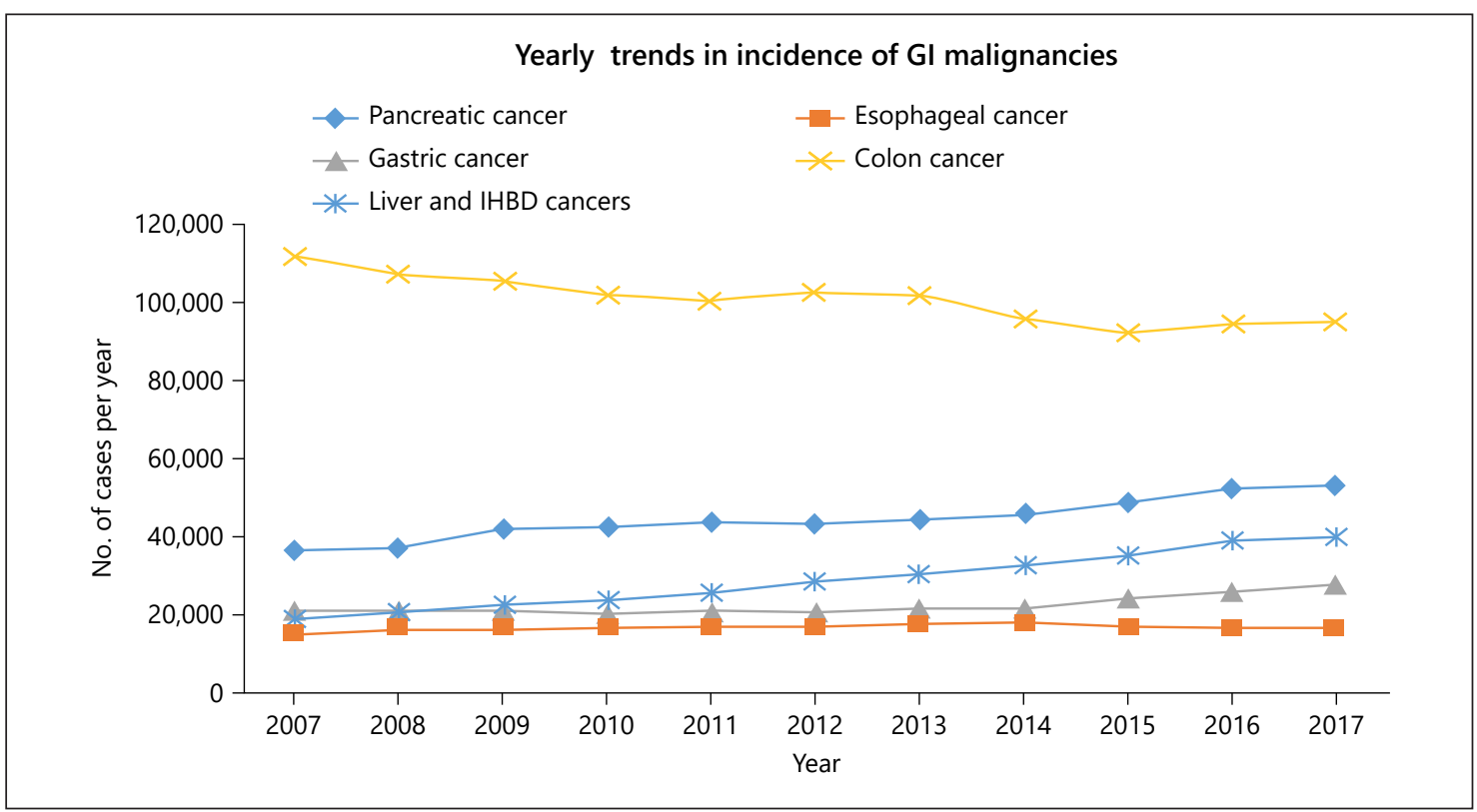

Fig. 2. Yearly trend in the incidence of GI malignancies by type, from 2007 to 2017 (derived from the annual cancer statistics reported by the American Cancer Society from 2007 to 2017 [24, 25]).

eficiaries and significantly lower Medicaid coverage. SVT with all types of GI malignancies were more likely to be treated at a teaching hospital located in urban areas. Esophageal and cholangiocarcinoma patients were treated in significantly higher numbers at a large-sized hospital ( $>450$ beds) than patients without GI malignancy. Baseline patient and hospital characteristics are summarized in Table 1. 


\section{In-Hospital Outcomes}

The Inpatient mortality rate was highest for SVT patients with gastric cancer $(12.1 \%, N=9)$, followed by patients with hepatic cancer $(7.6 \%, N=123)$ and cholangiocarcinoma $(6.7 \%, N=5)$. We performed univariate and multivariate logistic regression analysis to assess the baseline characteristics associated with increased odds of mortality (Table 2). Age (odds ratio: $1.03,95 \%$ confidence interval: $1.02-1.05)$, Charlson comorbidity index $(1.17,1.05-1.30)$, shock $(33.5,14.34-$ $65.77)$, and prolonged ventilation (64.5, 37.8-109.8) were associated with increased odds of inpatient mortality. On multivariate logistic regression analysis, patients with gastric and hepatic cancer had significantly higher odds of mortality than patients without GI cancer as shown in Table 3 (expanded results are available in online suppl. Table 2).

Among SVT associated with GI malignancies, mean total hospitalization charges were the highest in the pancreatic cancer group (USD 69,109 $\pm 22,347$ ) followed by hepatic cancer (USD 45,317 $\pm 2,957$ ) and colon cancer (USD 42,423 $\pm 5,658$ ). However, multivariate linear regression analysis showed patients with most malignant SVTs, including esophageal, hepatic, and cholangiocarcinoma or colorectal cancers had significantly lower total hospitalization charges than patients without GI malignancies. The mean length of stay was highest for patients with colon cancer $(5.69 \pm 0.46$ days) and pancreatic cancer related SVT (5.37 \pm 0.45 days). After accounting for confounders, hepatic and cholangiocarcinoma had significantly lower length of stays than patients with SVT without GI cancer. Patients with SVT and gastric, hepatic, pancreatic, or colon cancer had significantly higher odds of being discharged to a rehabilitation facility than patients with non-GI malignancy-related SVT. Primary inpatient outcomes are enlisted in Table 3.

Table 4 demonstrates the results of univariate logistic regression analysis comparing the rates of hospital complications seen in SVT associated with each major GI malignancy (hepatic, pancreatic, and colon cancer) with SVT associated with non-GI malignancies. SVT secondary to hepatic cancer was associated with significantly higher odds of hepatic encephalopathy, ascites, variceal bleeding, and requirement for blood transfusion but lower odds of mesenteric ischemia in comparison to non-GI cancer-associated SVTs. Pancreatic cancer-related SVT had higher odds of being complicated by ascites but lower odds of requiring blood transfusion and variceal bleeding as compared to non-GI cancer causes of SVT.

Splanchnic Vein Thrombosis in

Gastrointestinal Malignancies

\section{Discussion}

We present the first nationwide study on the hospitalizations for SVT in patients with GI malignancies, to provide data on the hospitalization rate, baseline inpatient characteristics, and hospital outcomes for SVT in the setting of GI malignancies. In our retrospective nationwide analysis, hepatic cancer accounted for half of all hospitalizations for SVT in digestive cancers. Pancreatic and colon cancer were the other two most common cancers. While the true incidence of SVT remains unclear, as many cases remain undiagnosed, the hospitalization rates are clearly higher for hepatic cancer associated SVT than the other GI tumors. A large, multi-hospital study analyzing PVT in patients with gastrointestinal tumors, excluding HCC, showed similar trends, with pancreatic adenocarcinoma and colorectal cancer accounting for about $75 \%$ of all cases [14]. PVTs are present in $10-40 \%$ of HCC at the time of diagnosis and are an adverse prognostic factor, noted to be associated with a higher rate of hospitalization than the other GI malignancies [15]. These data are possibly explained by a higher recognition of PVTs as a part of the natural history of cirrhosis and hepatocellular cancer. Screening for PVT is required as part of the current Barcelona Clinic Liver Cancer staging system as the prognosis for patients with HCC and PVT is much worse [8]. Meanwhile, the higher increase in trend of hospitalization in pancreatic adenocarcinomas for SVT-related malignancies is compatible with recent data, as published by Hicks et al. [16] and Afzal et al. [17], suggesting that SVT is not only a finding of more advanced stages of the disease but is in itself a predictor of mortality leading to more frequent admissions. The high incidence of such occurrence is explained by the pro-thrombotic and proangiogenic state associated with both malignancies that are necessary for tumor growth and metastasis. For pancreatic ductal adenocarcinoma, the high risk of thrombosis is caused by the pancreatic cancer itself, mainly by promoting the release of procoagulant factors, such as tissue factor and thrombin, inducing platelet aggregation via thrombin dependent and other mechanisms and creating an overall imbalance between procoagulant and anticoagulant factors $[9,18]$. The risk of thromboembolism in any of the venous vasculature is then further potentiated by the chemotherapeutic regimens, prompting the conduction of several studies like the CASSINI and the AVERT trials to reduce venous thromboembolism even in ambulatory patients undergoing systemic chemotherapy $[19,20]$. As for HCC, SVTs and especially PVT can be a consequence of the underlying cirrhosis, through a 
reduced portal flow due to architectural disarray in conjunction with local factors activating the clotting system [21]. It may also represent a direct invasion and growth of the tumor within the vasculature, which categorizes HCC patients with such complication as advanced stage $[22,23]$. Moreover, the incidence of pancreatic cancer and hepatic cancers in the USA have increased by $44 \%$ and $112 \%$, respectively, from 2007 to 2017 which justifies the rising trend in admissions for SVT related to these malignancies $[24,25]$. Interestingly, the opposite effect is seen when it comes to colon cancer. Although the incidence for colon cancer has decreased by $14 \%$ from 2007 to 2017, admissions related to SVT secondary to colon cancer have been steadily increasing [24, 25].

Interestingly, males were noted to be more likely to be hospitalized due to SVTs in hepatic and esophageal cancer, with females instead being more commonly admitted for SVT related to pancreatic cancer. According to Regnault et al. [14], the male gender was more at risk to develop PVT in all digestive cancer patients. In the same study, male sex also was noted to be a risk factor for gastrointestinal bleeding associated with portal hypertension (PHT), a known and frequent complication of PVT. We found a higher age and comorbidity index in patients with GI malignancies which could contribute to the worse prognosis in these patients. Analysis of hospital distribution showed that patients with GI malignancies and SVT were more likely to be treated at teaching hospitals, possibly due to the complexity of care required for such patients.

According to the results of the multivariate logistic regression analysis we conducted, patients with gastric and hepatic cancer had significantly higher odds of mortality than patients with SVT without GI cancer. Although SVT is a rare occurrence in gastric cancer, it seems to be associated with the highest inpatient mortality of $12.1 \%$. Our study also showed that the rates of variceal bleeding and blood transfusions were significantly higher in patients with hepatic cancer but significantly lower in patients with pancreatic cancer. This knowledge can help guide the role of anticoagulation in patients with GI malignancy complicated by visceral thrombosis. Data adjusted for confounders showed that esophageal, hepatic, cholangiocarcinoma, and colorectal cancers had significantly lower total hospitalization charges than patients without GI malignancies, with hepatic cancers and cholangiocarcinoma having a shorter duration of in-hospital stays. These findings can be explained with the severely debilitated status corresponding to these renowned aggressive cancers and a tendency to be less therapeutically aggres- sive given the overall poor prognosis. Besides, SVT hospitalizations occurring in patients without an obvious malignant cause tend to undergo extensive work-up to determine the etiology resulting in a longer and more expensive inpatient stay. Moreover, there are limited management options for malignancy-associated PVT, while also considering that some techniques, such as selective internal radiation therapy, can be executed as an outpatient.

The rationale behind anticoagulation in SVT is to prevent intestinal and splenic infarction via thrombus dissolution and recanalization, while also preventing PHT by interrupting the progression to cavernous transformation of the portal vein [18]. As of now, the mainstay of treatment of thrombosis of the splanchnic veins in the cancer setting is low molecular weight heparin or, less commonly, unfractionated heparin. While vitamin $\mathrm{K}$ antagonists, bridged with low molecular weight heparin are suggested for the majority of patients with SVTs, evidence points to them being less efficient and safe in patients with cancer, hepatic disease, or low platelet count $[26,27]$. An increasingly used option is represented by direct oral anticoagulants, an alternative made attractive due to their ease of use. Lack of data investigating their efficacy and safety, however, limits their use and hinders their inclusion as a standard of care in this specific population. The present-day therapeutic strategies are extrapolated from guidelines made by the American College of Chest Physicians despite the fact that they are not specifically targeted towards SVT [28]. Moreover, there are no randomized clinical trials regarding anticoagulation in these patients, and most of the data are inferred from observational studies that do not take into account the different etiologies of SVT and consequently dissimilar natural courses. The retrospective analysis conducted by Acuna-Villaorduna et al. [29] on 698 patients revealed significant differences in outcomes, with a much higher rate of PVT progression and venous thromboembolism recurrence in the malignancy group compared to the patients with cirrhosis or acute abdominal inflammatory conditions. Furthermore, the incidence of thrombotic outcomes was similar between patients with symptomatic SVT and those with incidental SVT. This finding is of particular importance given that the current guidelines recommend anticoagulation only for patients with symptomatic SVT while suggesting a case by case approach in incidental SVTs. Since we included admissions for SVT as the primary diagnosis, it is more likely to be symptomatic than incidental. We showed that advanced age and higher comorbidity index increased the odds of inpatient 
mortality, factors which are also associated with increased bleeding risk. A recent study does support the notion that patients with incidentally detected SVT should receive anticoagulation, while also underlining that the mortality associated with major bleeding during the course of anticoagulation may outweigh the mortality associated with VTE recurrences, advising caution in such a delicate equilibrium [30].

Our analysis also showed a fourfold increase in the hospitalization rate in GI malignancies during the 11year study period. This rapid increase is a possible result of an increased detection of SVT due to increased awareness of the former as an anticipated complication as well as an actual rise in incidence in the context of prolonged survival due to diagnostic and therapeutic advancements. This setting appears favorable to broaden the knowledge of this subgroup of patients, with a focus on complications associated with PVT-mainly PHT and variceal bleeding.

This study carries limitations intrinsic to its observational nature which may reduce the accuracy of the data. Sampling bias was averted by using previously validated ICD 10 codes to capture the right study sample. Second, because of the administrative nature of our database, we could not define the size and the location subtype of the PVT (truncal or segmental). Furthermore, we cannot differentiate between incidental or symptomatic SVT as well as an actual thrombus versus tumor thrombus. Also, since the actual cause of death is not captured in the NIS, we have reported the total all-cause mortality without delineating the actual cause. Lastly, we could not identify the two other types of SVT, that is, splenic vein and mesenteric vein thrombosis, given that they currently do not have a specific ICD 9 or ICD 10 codes. It remains unequivocal, however, that more in-depth knowledge of this ambiguous condition, with its heterogeneous presentations and complications, including not only PHT but also bowel ischemia, will possibly lead to an increased burden in terms of hospitalization charges and a rise in hospitalization rates-such as already observed in our study. Additional complications related to treatment can only increase such burden and therefore merit further studies, encompassing the possibility of validating the use of direct oral anticoagulants in such settings.

\section{Statement of Ethics}

This paper is exempt from Ethical Committee approval as it does not contain any studies involving human participants or animals. The database used for the study only includes de-identified data.

\section{Conflict of Interest Statement}

All the listed authors declare that they have no conflict of interest.

\section{Funding Sources}

The authors declare that there was no grant or financial support received whatsoever for the research conducted by us and writing of this article.

\section{Author Contributions}

Dr. Handa and Dr. Gupta contributed equally to this manuscript and should be considered co-first authors. Dr. Handa conceived the study idea and took the lead in writing the manuscript. Dr. Gupta devised the statistical analysis plan and wrote the statistical code. Dr. Sterpi authored the discussion section of the manuscript. Dr. Khan ran the statistical tests. Dr. Hoskote performed background literature search. Dr. Kasi was our faculty mentor who revised and edited the final manuscript. All authors provided critical feedback and helped shape the research analysis and manuscript.

\section{References}

1 Smalberg JH, Kruip MJ, Janssen HL, Rijken DC, Leebeek FW, de Maat MP. Hypercoagulability and hypofibrinolysis and risk of deep vein thrombosis and splanchnic vein thrombosis: similarities and differences. Arterioscler Thromb Vasc Biol. 2011;31(3):485-93.

2 Rajani R, Melin T, Björnsson E, Broomé U, Sangfelt P, Danielsson A, et al. Budd-Chiari syndrome in Sweden: epidemiology, clinical characteristics and survival: an 18-year experience. Liver Int. 2009;29(2):253-9.
3 Ogren M, Bergqvist D, Björck M, Acosta S, Eriksson H, Sternby NH. Portal vein thrombosis: prevalence, patient characteristics and lifetime risk: a population study based on 23,796 consecutive autopsies. World J Gastroenterol. 2006;12(13):2115-9.

4 Serrano PE, Parpia S, Linkins LA, Elit L, Simunovic M, Ruo L, et al. Venous thromboembolic events following major pelvic and abdominal surgeries for cancer: a prospective cohort study. Ann Surg Oncol. 2018;25(11): 3214-21.
5 Siegel RL, Miller KD, Goding Sauer A, Fedewa SA, Butterly LF, Anderson JC, et al. Colorectal cancer statistics, 2020. CA Cancer J Clin. 2020;70(3):145-64.

6 Manzano-Robleda Mdel C, Barranco-Fragoso $\mathrm{B}$, Uribe M, Méndez-Sánchez N. Portal vein thrombosis: what is new? Ann Hepatol. 2015;14:20-7.
Splanchnic Vein Thrombosis in Gastrointestinal Malignancies
Gastrointest Tumors 2021;8:71-80 DOI: $10.1159 / 000513368$ 
7 Cerrito L, Annicchiarico BE, Iezzi R, Gasbarrini A, Pompili M, Ponziani FR. Treatment of hepatocellular carcinoma in patients with portal vein tumor thrombosis: beyond the known frontiers. World J Gastroenterol. 2019;25(31):4360-82.

8 Chew HK, Wun T, Harvey D, Zhou H, White $\mathrm{RH}$. Incidence of venous thromboembolism and its effect on survival among patients with common cancers. Arch Intern Med. 2006; 166(4):458-64.

9 Khorana AA, Fine RL. Pancreatic cancer and thromboembolic disease. Lancet Oncol. 2004; 5(11):655-63.

10 Thodiyil PA, Kakkar AK. Variation in relative risk of venous thromboembolism in different cancers. Thromb Haemost. 2002;87(6):10767.

11 Menapace LA, Peterson DR, Berry A, Sousou T, Khorana AA. Symptomatic and incidental thromboembolism are both associated with mortality in pancreatic cancer. Thromb Haemost. 2011;106(2):371-8.

12 Lee J-C, Cho J, Park Y, Ro YS, Choi H. Venous thromboembolism in patients with pancreatic cancer: incidence and effect on survival in east Asian ethnic groups. J Clin Oncol. 2013; 31(4_suppl):151.

13 Gupta K, Khan A, Goyal H, Cal N, Hans B, Martins T. Weekend admissions with ascites are associated with delayed paracentesis: a nationwide analysis of the "weekend effect". Ann Hepatol. 2020;19(5):523-9.

14 Regnault H, Emambux S, Lecomte T, Doat S, Dhooge M, Besson M, et al. Clinical outcome of portal vein thrombosis in patients with digestive cancers: a large AGEO multicenter study. Dig Liver Dis. 2018;50(3):285-90.
15 Quirk M, Kim YH, Saab S, Lee EW. Management of hepatocellular carcinoma with portal vein thrombosis. World J Gastroenterol. 2015;21(12):3462-71.

16 Hicks AM, DeRosa A, Raj M, Do R, Yu KH, Lowery MA, et al. Visceral thromboses in pancreas adenocarcinoma: systematic review. Clin Colorectal Cancer. 2018;17(2):e207-16.

17 Afzal A, Suhong L, Gage BF, Schoen MW, Carson K, Thomas T, et al. Splanchnic vein thrombosis predicts worse survival in patients with advanced pancreatic cancer. Thromb Res. 2020;185:125-31.

18 Heinmöller E, Schropp T, Kisker O, Simon B, Seitz R, Weinel RJ. Tumor cell-induced platelet aggregation in vitro by human pancreatic cancer cell lines. Scand J Gastroenterol. 1995; 30(10):1008-16.

19 Khorana AA, Soff GA, Kakkar AK, VadhanRaj S, Riess H, Wun T, et al. Rivaroxaban for thromboprophylaxis in high-risk ambulatory patients with cancer. N Engl J Med. 2019; 380(8):720-8

20 Carrier M, Abou-Nassar K, Mallick R, Tagalakis V, Shivakumar S, Schattner A, et al. Apixaban to prevent venous thromboembolism in patients with cancer. N Engl J Med. 2019; 380(8):711-9.

21 Kinjo N, Kawanaka H, Akahoshi T, Matsumoto Y, Kamori M, Nagao Y, et al. Portal vein thrombosis in liver cirrhosis. World J Hepatol. 2014;6(2):64-71.

22 Jiang JF, Lao YC, Yuan BH, Yin J, Liu X, Chen $\mathrm{L}$, et al. Treatment of hepatocellular carcinoma with portal vein tumor thrombus: advances and challenges. Oncotarget. 2017;8(20): 33911-21.
23 Liu PH, Huo TI, Miksad RA. Hepatocellular carcinoma with portal vein tumor involvement: best management strategies. Semin Liver Dis. 2018;38(3):242-51.

24 Jemal A, Siegel R, Ward E, Murray T, Xu J, Thun MJ. Cancer statistics, 2007. CA Cancer J Clin. 2007;57(1):43-66.

25 Siegel RL, Miller KD, Jemal A. Cancer statistics, 2017. CA Cancer J Clin. 2017;67(1):7-30.

26 Lee AY, Levine MN, Baker RI, Bowden C, Kakkar AK, Prins M, et al. Low-molecularweight heparin versus a coumarin for the prevention of recurrent venous thromboembolism in patients with cancer. $\mathrm{N}$ Engl J Med. 2003;349(2):146-53.

27 Kearon C, Akl EA, Comerota AJ, P, H, SZ, et al. Antithrombotic therapy for VTE disease: antithrombotic therapy and prevention of thrombosis, 9th ed: American College of Chest Physicians evidence-based clinical practice guidelines [published correction appears in Chest. 2012 Dec;142(6):1698-1704]. Chest. 2012;141(2 Suppl):e419S-96S.

28 Kearon C, Akl EA, Ornelas J, A, D, H, et al. Antithrombotic therapy for VTE disease: CHEST guideline and expert panel report [published correction appears in Chest. 2016 Oct;150(4):988]. Chest. 2016;149(2):315-52.

29 Acuna-Villaorduna A, Tran V, Gonzalez-Lugo JD, Azimi-Nekoo E, Billett HH. Natural history and clinical outcomes in patients with portal vein thrombosis by etiology: a retrospective cohort study. Thromb Res. 2019;174: $137-40$.

30 Tufano A, Ageno W, Di Micco P, Niglio A, Rosa V, Ballaz A, et al. Outcomes during anticoagulation in patients with symptomatic vs. incidental splanchnic vein thrombosis. Thromb Res. 2018;164:69-74. 